\title{
A study of pedestrian-vehicle accidents in Iran in 2013
}

\author{
Afshari M, MSc ${ }^{1 *}$, Khazaei S, MSc${ }^{2,3}$, Mohammadian M, $\mathrm{MSc}^{4}$, Mohammadian Hafshejani \\ $\mathrm{A}, \mathrm{MSc}^{5}$ \\ 1- MSc, PhD Student in Health Education and Health Promotion, School of Public Health, Hamadan University of Medical \\ Sciences, Hamadan, Iran. 2- MSc, PhD Candidate in Epidemiology, Dept. of Epidemiology and Biostatistics, School of \\ Public Health, Tehran University of Medical Sciences, Tehran, Iran. 3- MSc, PhD Candidate in Epidemiology, Deputy of \\ Health, Hamadan University of Medical Sciences, Hamadan, Iran. 4- MSC in Epidemiology, Social Development and \\ Health Promotion Research Center, Gonabad University of Medical Sciences, Gonabad, Iran. 5- MSc, PhD Candidate in \\ Epidemiology, Social Determinants in Health Promotion Research Center, Hormozgan University of Medical Sciences, \\ Bandar Abbas, Iran.
}

\begin{abstract}
Received: July 2016, Accepted: October 2016

Background: In recent decades, due to the increase in users of roads and inadequate attention to the creation and development of appropriate passageways for traffic, there has been a growing trend in traffic accidents and casualties caused by them. The traffic accidents among pedestrians to take necessary measures in a timely fashion should be well studied. This study was conducted with the goal of survey traffic accidents among pedestrians in 2014.

Materials and Methods: This cross-sectional study was conducted using data from the National Accidents Record Program regarding 2013. In this study, all traffic accidents in which the injured person was referred to a public or private hospital in the country to receive health services or was transferred to the hospital by EMS 115 and received remedial measures on an outpatient or inpatient basis in all provinces were considered. The incidence of pedestrian-vehicle accidents was calculated per ten thousand. Map GIS software package was used for the mapping of occurrence of events and Microsoft EXCEL and Stata software were used for data analysis.

Results: In this study, the 82066 pedestrians who were injured in road accidents consisted of a total of 53240 men and 28826 women. The rate of traffic accidents was higher in men than in women $(64.87 \%$ and $35.13 \%$, respectively) and the majority of individuals affected were in the age group of 20 to 24 years. Most pedestrian-vehicle accidents had occurred on the street (89.8\%).

Conclusion: Pedestrians are at risk of the irreversible outcomes of traffic accidents; therefore, effective and regular training on the prevention of injury and secure environment is essential to reduce injuries.
\end{abstract}

Keywords: Accidents, Traffic, Injury, Pedestrians

\section{Introduction}

In recent decades, with the increase in users of roads and inadequate attention to the creation and development of appropriate passageways for traffic, there has been a growing trend in traffic accidents and casualties caused by them (1). Injuries caused by car accidents are one of the major problems in the world that threaten the human health (2). Nearly 2.1 million individuals lose their lives due to traffic accidents each year, and more than 50 million individuals suffer some degrees of damages (3). It has been estimated that this amount increased to $65 \%$ in the whole world and nearly $80 \%$ in developing countries from 2000 to 2020 (4). Many of those who are at risk of damages caused by traffic accidents are pedestrians and do not have vehicles (5). In developed countries, 30 to 40 killed or injured per hundred thousand pedestrians are due to car accidents each year, and this problem is increasing rapidly in developing countries (6).

\footnotetext{
* Corresponding author: Maryam Afshari, School of Public Health, Hamadan University of Medical Sciences, Hamadan, Iran.

Email: afshari_m20@yahoo.com
} 
Almost a third of vehicles ply on different routes on a daily basis in developing countries, but the majority of traffic accidents involve pedestrian in these countries (7). The most important factors that increase injuries caused by traffic accidents particularly among pedestrians in Iran include an increase in the number of vehicles, inadequate and insufficient infrastructures of roads and health sector, unfavorable status of traffic control, use of non-standard vehicles, poor safety on roads and streets, and lack of traffic culture for pedestrians $(7,8)$.

Today, with the increase in the production and development of vehicles on the one hand and urbanization population growth on the other hand, attention to issues of pedestrians particularly in terms of security is necessary (9). Pedestrian injuries cause many issues in the society such as social problems and economic costs, and intensify the existing problems within the society (10). It is clear that continuation of the current growth in injuries caused by accidents among pedestrians and failure to adopt desirable and appropriate predictive and preventative methods can be a potential threat to the population of the country and waste many material and immaterial resources (11).

Given that our country is also among developing countries and the number of vehicles is rapidly increasing in this country, traffic problems especially for pedestrians will be the most important issues that threaten public health and increase unnecessary costs within the society, particularly for pedestrians (12). In a study which was conducted on secure traffic behaviors among students by Ramezankhani et al. in 2013 based on the Theory of Planned Behavior, the results showed that training can affect the secure behaviors of students in crossing the street (10). Similarly, in another study which was conducted on pedestrians in Tehran (Iran) by Holakouee and Moradi in 2007, the results showed that the performance of pedestrians regarding traffic regulations is poor in Tehran (11). Maki and Kajzer reviewed pedestrian accident cases in Japan from 1995 to 1998 (13). They found that $22 \%$ of serious injuries and $64 \%$ of fatal injuries were head injuries. This finding indicated that the odds to be fatally injured were 6.3 times as high when sustaining a head injury relative to when sustaining other injuries (13). Moreover, the results of a study by Yao et al. showed that a sizeable proportion of adult pedestrians involved in vehicle-versus-pedestrian accidents suffer head injuries, some of which can lead to lifelong disability or even death (14).

Therefore, controlling and moderating these issues as well as promoting desirable methods and appropriate culture-making including the culture of not responding to the cell phone while driving, not eating or drinking while driving, driving with reliable speed, attention to pedestrian crossings and crosswalks, attention to traffic lights, and other necessary precautions are related to the epidemiological characteristics of accidents (15). Thus, the best and most economical way of reducing and controlling the occurrence of injuries caused by traffic accidents, particularly in pedestrians, can be adopted. Therefore, this study was conducted with the goal of survey traffic accidents among pedestrians in 2014.

\section{Material and Methods}

This cross-sectional, descriptive, and analytical study was conducted using data of the National Accidents Record Program related to 2013 in Iran. In this study, all traffic accidents in which the injured person was referred to a public or private hospital in the country to receive health services or was transferred to the hospital by EMS 115 and received remedial measures on an outpatient or inpatient basis in all provinces were considered. Other accidents that resulted in deaths at the scene of the crash or individuals who did not have to go to the hospital due to mild injuries were not considered in the study because no information was available on them. In this study, the required information were 
extract from patients' emergency care report forms and medical history information that were obtained by nurses and physicians using the National Accidents Record Program. This information is usually extracted from patient records by a medical records expert and is entered into the National Accidents Record Program software. This information is completed by hospitals and is transferred to provincial health centers periodically and information related to all of the cities of a province is collected for each year (16). In this study, hospital incidence rate of pedestrianvehicle accidents is calculated and presented on the basis of per ten thousand people. To calculate the incidence rate in each province, the population of that province in 2013 was considered. Mapping the occurrence of accidents was conducted using ArcGIS software (version 10, Esri, Redlands, CA, USA) and a map of low and high risk areas of the country was presented.

In addition, the risk ratio of occurrence of traffic accidents on the basis of sex (male to female) was calculated and presented with 95\% confidence intervals. Microsoft EXCEL software (2010, Microsoft Corp., Redmond, WA, USA) and Stata software (version 11, StataCorp LP, TX, USA) were used to analyze the data. In this study, the significance level was considered as less than 0.05 .

Table 1: The incidence rate and sex ratio of pedestrian-vehicle accidents in each province in 2013 (82066 individuals)

\begin{tabular}{|c|c|c|c|c|c|c|c|}
\hline Province & $\begin{array}{c}\text { Incidence rate } \\
\text { per } 10000\end{array}$ & Men & Women & $\begin{array}{c}\text { Risk ratio } \\
\text { (M/W) }\end{array}$ & $\begin{array}{l}\text { Upper } \\
\text { bound }\end{array}$ & $\begin{array}{l}\text { Lower } \\
\text { bound }\end{array}$ & $\mathbf{P}$ \\
\hline Azarbaijan Sharghi & 9.21 & 2152 & 1268 & 1.7 & 1.77 & 1.64 & 0.001 \\
\hline Azarbaijan Gharbi & 13.80 & 2844 & 1317 & 2.2 & 2.27 & 2.14 & 0.001 \\
\hline Ardabil & 3.50 & 312 & 141 & 2.1 & 2.17 & 2.04 & 0.001 \\
\hline Isfahan & 12.02 & 3377 & 2325 & 1.5 & 1.57 & 1.44 & 0.001 \\
\hline Alborz & 14.52 & 2149 & 1192 & 1.8 & 1.87 & 1.74 & 0.001 \\
\hline Ilam & 8.33 & 287 & 176 & 1.6 & 1.67 & 1.54 & 0.001 \\
\hline Bosheher & 3.76 & 229 & 145 & 1.6 & 1.67 & 1.54 & 0.001 \\
\hline Tehran & 11.23 & 9954 & 4985 & 2 & 2.07 & 1.94 & 0.001 \\
\hline $\begin{array}{c}\text { Chaharmahal and } \\
\text { Bakhtiari }\end{array}$ & 1.61 & 102 & 50 & 2 & 2.07 & 1.94 & 0.001 \\
\hline Khorasan Jonobi & 6.62 & 227 & 224 & 1.2 & 1.27 & 1.14 & 0.001 \\
\hline Khorasan Shomali & 19.30 & 1041 & 632 & 1.6 & 1.67 & 1.54 & 0.001 \\
\hline Khorasan Razavi & 16.92 & 6865 & 3603 & 1.9 & 1.97 & 1.54 & 0.001 \\
\hline Khozestan & 11.45 & 3863 & 1745 & 2.2 & 2.27 & 2.14 & 0.001 \\
\hline Zanjan & 3.28 & 226 & 116 & 1.9 & 1.97 & 1.54 & 0.001 \\
\hline Semnan & 14.88 & 521 & 411 & 1.3 & 1.37 & 1.24 & 0.001 \\
\hline $\begin{array}{c}\text { Sistan and } \\
\text { Balochestan }\end{array}$ & 3.94 & 761 & 250 & 3 & 3.07 & 2.94 & 0.001 \\
\hline Fars & 6.18 & 1805 & 1094 & 1.6 & 1.67 & 1.54 & 0.001 \\
\hline Ghazvin & 8.49 & 665 & 365 & 1.8 & 1.87 & 1.74 & 0.001 \\
\hline Ghom & 19.45 & 1433 & 927 & 1.5 & 1.57 & 1.44 & 0.001 \\
\hline Kordestan & 4.73 & 473 & 276 & 1.7 & 1.77 & 1.64 & 0.001 \\
\hline Kerman & 12.84 & 2059 & 1097 & 1.9 & 1.97 & 1.84 & 0.001 \\
\hline Kermanshah & 31.37 & 4225 & 1943 & 2.2 & 2.27 & 2.14 & 0.001 \\
\hline $\begin{array}{c}\text { Kohgiluyeh and } \\
\text { Boyer-Ahmad }\end{array}$ & 5.34 & 217 & 158 & 1.4 & 1.47 & 1.34 & 0.001 \\
\hline Golestan & 13.06 & 1437 & 857 & 1.7 & 1.77 & 1.64 & 0.001 \\
\hline Gilan & 10.20 & 1647 & 674 & 2.4 & 2.47 & 2.34 & 0.001 \\
\hline Lorestan & 6.52 & 788 & 412 & 1.9 & 1.97 & 1.84 & 0.001 \\
\hline Mazandaran & 11.57 & 2084 & 1473 & 1 & 1.07 & 0.94 & 0.254 \\
\hline Markazi & 3.34 & 325 & 160 & 1.8 & 1.87 & 1.74 & 0.001 \\
\hline Hormozgan & 3.23 & 325 & 160 & 2 & 2.07 & 1.94 & 0.001 \\
\hline Hamadan & 2.56 & 254 & 208 & 1.2 & 1.27 & 1.14 & 0.001 \\
\hline Yazd & 10.22 & 593 & 442 & 1.3 & 1.37 & 1.24 & 0.001 \\
\hline
\end{tabular}




\section{Results}

In this study, the 82066 pedestrians who suffered from a traffic accident and its consequent injuries during 2013 included $53240(64.87 \%)$ men and 28826 (35.13\%) women.

The study results regarding pedestrian-vehicle accidents presented in table 1 show that the highest frequency of injured individuals was related to men $(64.87 \%)$. The sex ratio of injured pedestrians among men was higher than women in all provinces of Iran except Mazandaran (Iran) in which the sex ratio was equal between injured men and women. The incidence rate of pedestrian-vehicle accidents

per 10000 was highest in the province of Kermanshah (31.37\%) and lowest in the provinces of Chaharmahal and Bakhtiari (1.61\%), and Hamedan (Iran) (2.56\%), respectively.

The results of table 2 show that the highest frequency of the location of pedestrian-vehicle accidents occurrence was related to the alleys and streets (89.8\%). Furthermore, according to sex, the majority of accidents had occurred in men (88.6\%) and women (91.5\%) in the alleys and streets, respectively, and the least accidents had occurred in the school in both sexes $(0.1 \%)$.

Table 2: Frequency of the location of pedestrian-vehicle accidents occurrence (82066 individuals)

\begin{tabular}{lccc}
\hline Location of traffic accidents & Men & Women & Total \\
\cline { 2 - 4 } & Number $(\boldsymbol{\%})$ & Number $(\boldsymbol{\%})$ & Number $(\%)$ \\
\hline House yard and surroundings & $160(0.2)$ & $113(0.2)$ & $273(0.3)$ \\
School and education facilities & $28(0.1)$ & $8(0.1)$ & $36(0.1)$ \\
Public areas & $304(0.6)$ & $173(0.5)$ & $477(0.6)$ \\
Sport and recreation areas & $112(0.2)$ & $63(0.2)$ & $175(0.2)$ \\
Alleys and streets & $47393(88.6)$ & $26364(91.5)$ & $73757(89.8)$ \\
Highways and roads & $4611(9.0)$ & $1812(6.0)$ & $6423(7.8)$ \\
Around the workplace* & $122(0.2)$ & $14(0.4)$ & $136(0.1)$ \\
Other areas** & $48(0.1)$ & $25(0.1)$ & $73(0.1)$ \\
Unknown*** & $533(1.0)$ & $284(1.0)$ & $817(1.0)$ \\
* Occupational accidents in the workplace in collisions with cars & & \\
** Locations other than the locations listed above & & \\
*** Location of the accident of pedestrians who were not identified & &
\end{tabular}

Moreover, the frequency of pedestrian-vehicle accidents in terms of age group in the country in 2013 is illustrated in figure 1. The majority of pedestrian-vehicle accidents in both sexes had occurred in the age group of 20 to 24 years, and the least number of pedestrianvehicle accidents in both sexes had occurred in the age group of 85 years.

Figure 2 shows the incidence rate of pedestrian-vehicle accidents in the country. In the provinces that are specified with the color white, the incidence rate of pedestrian-vehicle accidents ranged between 1.61 and 4.73 per 10000 people. They have a better situation than the provinces that are specified with yellow and red colors (incidence rate of pedestrian-vehicle accidents: 13.81 to 31.37 per 10000 people). 


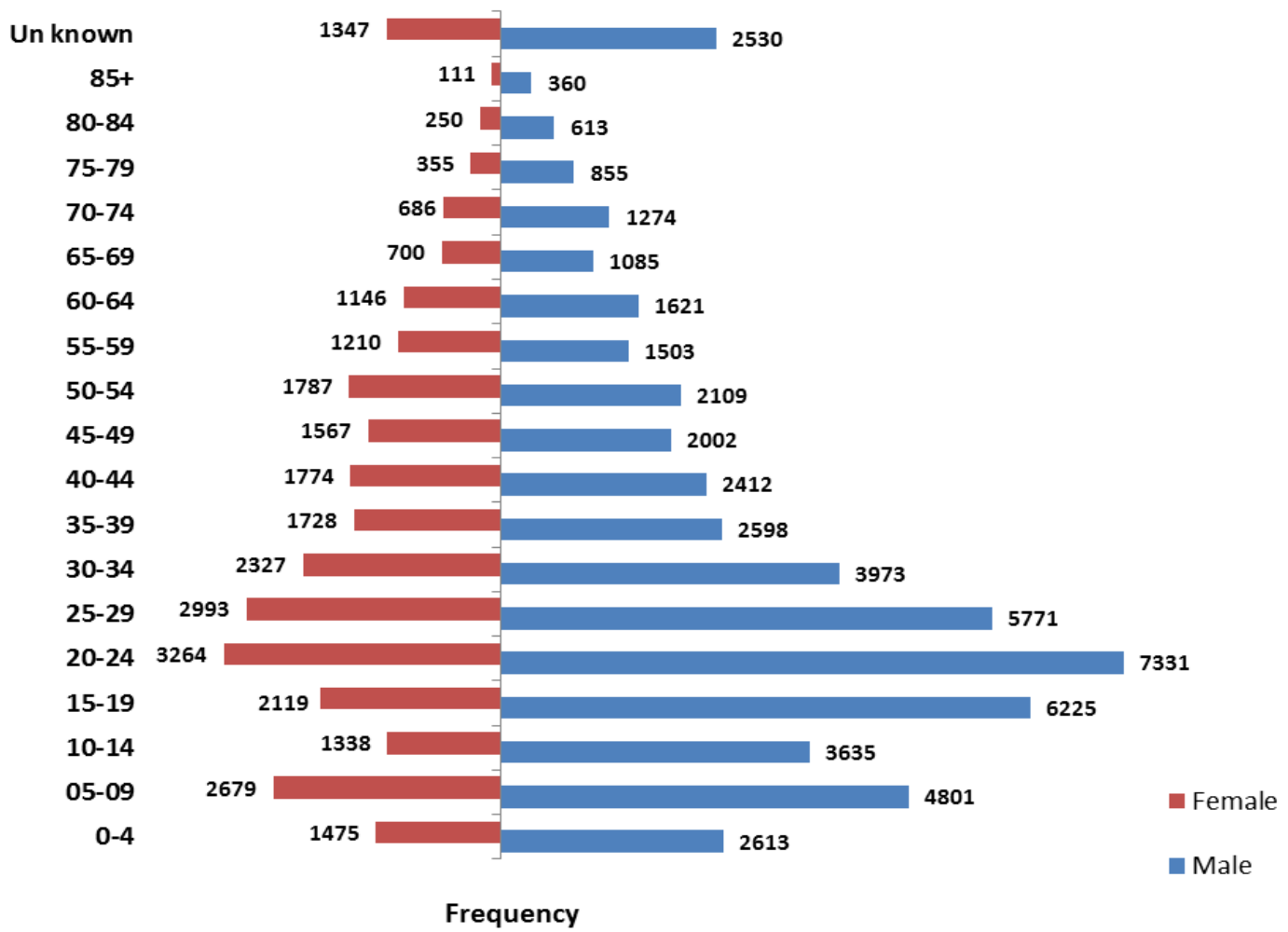

Figure 1: The frequency of pedestrian-vehicle accidents in terms of age group in the country in 2013 (82066 individuals)

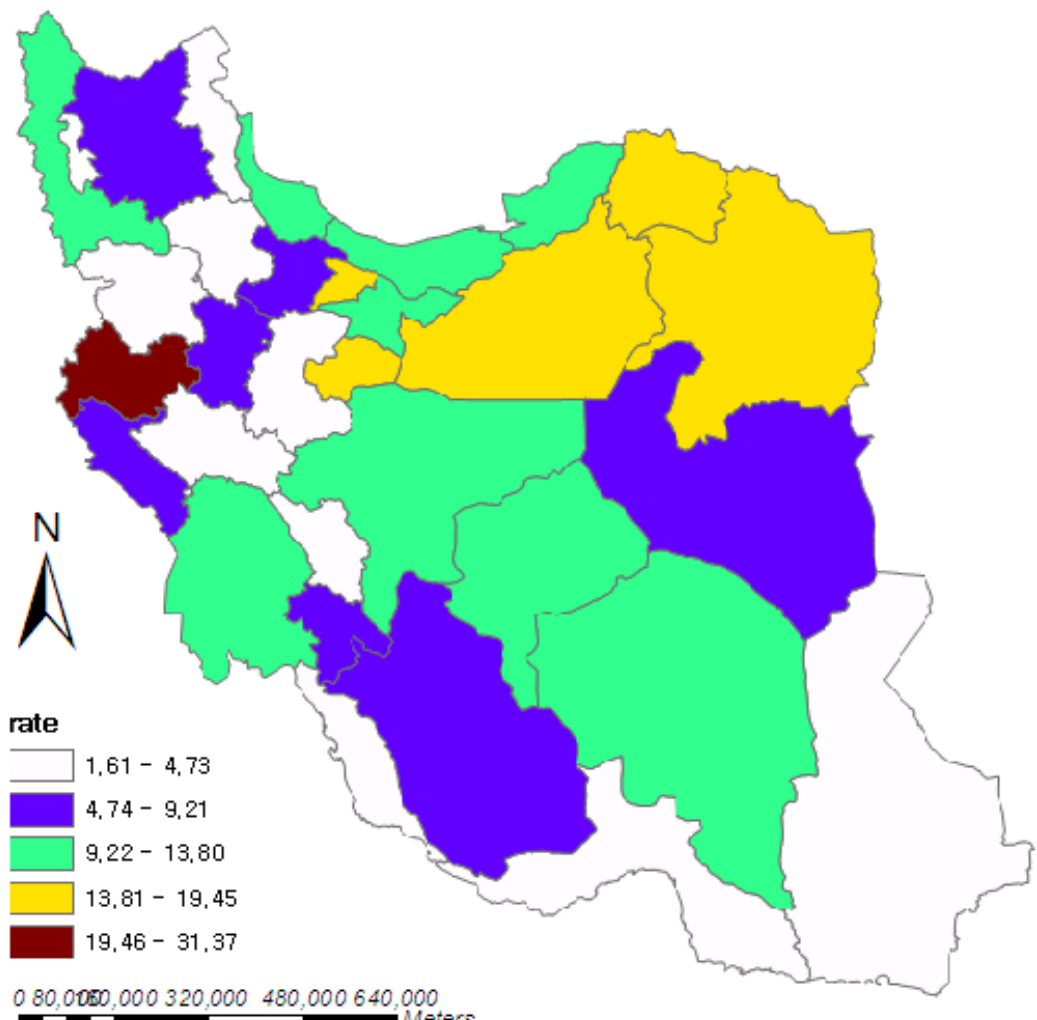

Figure 2: Incidence rate of pedestrian-vehicle accidents in the country (82066 individuals) 


\section{Discussion}

This study was conducted with the goal of determining the rate of pedestrian-vehicle accidents in 2013. As mentioned, in this study, the frequency of injuries in men was higher than women. In addition, the sex ratio was greater than 1 in all provinces apart from Mazandaran, which indicates a higher proportion of men in these accidents. The average amount of fatal lesions in men is $24 \%$ more than women according to statistics from the World Health Organization (WHO) (17). Moreover, the results of the study by Nazari et al. on the mortality rate and severity of injuries caused by traffic accidents in patients admitted to hospital in Amol (Iran) in 2011 showed that men were injured more than women (18). Investigation of injured people in terms of sex distribution in other studies, including the studies by Menon et al. and Dandona et al., show that the majority of injured people were men (19, 20). Furthermore, Maki and Kajzer reviewed pedestrian accident cases in Japan from 1995 to 1998 and found that men were injured more than women (13).

This can be due to working conditions, high working hours outside the home, and more physical activity among men. The majority of injured people in the study were of the age group of 20 to 24 years. In addition, several conducted studies have confirmed that traffic accidents occur in young age groups $(19,21$, 22). This study showed that most injured people are those who are at working ages. The high rate of accidents in this age group is probably due to Iran's young population as well as the educational, occupational, and physical conditions of this age group who spend more time outdoors. The highest frequency of the location of pedestrian-vehicle accidents occurrence was related to the alleys and streets. This result was almost consistent with that of the study conducted by Mobaleghi et al. on mortality caused by traffic accidents at the hospital in Sanandaj (Iran) in 2002 (12). In their study, almost $2.33 \%$ of those who had been referred to the hospital were pedestrians
(12). The results of other studies, including the study by Peden et al. on traffic accidents according to the WHO in 2004 as well as the study by Karbakhsh et al. in 2004, showed that suburban accidents have particular importance $(7,22)$. However, in other areas of the world, urban accidents had greater importance according to the WHO (23). The results of the study of WHO in 2001 showed that $55-77 \%$ of accidents had occurred in urban areas (23). The inconsistency between the results of this study and other studies $(7,22)$ is probably due to an increase in cars in the city, lack of suitability of the existing roads, and the poor safety culture.

Incidence rate of pedestrian-vehicle accidents in the country shows that the provinces specified with red and yellow colors in figure 2 had the most pedestrian-vehicle accidents. This can be because most cities which have higher incidence rate are religious cities with higher commuting rates, and are significant in terms of structural, economic, and political situations, and the higher commuting rate leads to problems such as pedestrian accidents. Furthermore, accident analysis revealed that the windshield and its surrounding frames were the main sources of head injury for adult pedestrians (14). The use of information from patients' medical records was the limitation of this study, and $100 \%$ use of all available evidence for pedestrian-vehicle accidents was the advantage of this study.

\section{Conclusion}

Given that the injuries had occurred in young age groups, being at risk of injuries caused by traffic accidents as well as higher consequent mortality and disability rates in this age group. Therefore, young people and authorities should be aware of the possible consequences resulting from injuries caused by traffic accidents and take measures to avoid and prevent these accidents. According to the abovementioned issues, the prediction and prevention of injuries is very essential. It seems that in prophylactic and intervention 
programs, causal factors of pedestrian-vehicle accidents and the ways to prevent them should be fully distinguished. Ultimately, the results obtained can provide planners and health authorities with appropriate strategies in the studied field in order to design effective programs.

\section{Acknowledgement}

We would like to thank all those who participated in this project and helped us in performing this project.

\section{Conflict of interests: None declared}

\section{References}

1- Naghavi M. Transition in health status in the Islamic Republic of Iran. Iranian Journal of Epidemiology 2006; 2(1-2):45-57.

2- Roberts I, Mohan D, Abbasi K. War on the roads. BMJ 2002; 324(7346):1107-8.

3- Nantulya VM, Reich MR. The neglected epidemic: road traffic injuries in developing countries. BMJ 2002; 324(7346):1139-41.

4- Naghavi M, Abolhassani F, Pourmalek F, Lakeh M, Jafari N, Vaseghi S, et al. The burden of disease and injury in Iran 2003. Popul Health Metr 2009; 7;9.

5- Farchi S, Molino N, Giorgi Rossi P, Borgia P, Krzyzanowski M, Dalbokova D, et al. Defining a common set of indicators to monitor road accidents in the European Union. BMC Public Health 2006; 6:183.

6- Mock C, Kobusingye O, Anh le V, Afukaar F, Arreola-Risa C. Human resources for the control of road traffic injury. Bull Word Healh Organ 2005; 83(4):294-300.

7- Peden M, Scurfield R, Sleet D, Mohan D, Hyder AA, Jarawan E, et al, editors. World report on road traffic injury prevention. Genava: World Health Organization; 2004. P.3-7.

8- Abdali H, Memarzadeh M. Evaluation of injury severity in traumatized patients at $\mathrm{AL}$ Zahra Medical Acadamic Center. Journal of Military Medicine 2003; 4(4):247-50.

9- Nantulya VM, Reich MR. The neglected epidemic: road traffic injuries in developing countries. Br Med J 2002; 324(7346):1139-41.

10- Ramezankhani A, Khalafeh Nilsaz M, Dehdari T, Soori H, Tavasoli E, Khezli M, etal. Effects of an educational intervention based on planned behavior theory in promoting safe behaviors crossing the street in students. Journal of Health System Research 2014; 9(14):2000-10.

11- Holakoee Naeeni K, Moradi A. Knowledge, attitude and performance pedestrians on traffic regulations in the area through the main intersection in Tehran. Payesh 2007; 6(1):2735 .

12- Mobaleghi J, Molanaee N. Road accident mortality and morbidity in Besat hospital accident ward. Scientific Journal of Kurdistan University of Medical Sciences 2002; 6(4):2833.

13- Maki T, Kajzer J, Mizuno K, Sekine Y. Comparative analysis of vehicle-bicyclist and vehicle-pedestrian accidents in Japan. Accid Anal Prev 2003; 35(6):927-40.

14- Yao J, Yang J, Otte D. Investigation of head injuries by reconstructions of real-world vehicle-versus-adult-pedestrian accidents. Saf Sci 2008; 46(7):1103-14.

15- Khazaie S, Mohammadian Hafshejani A, Mohammadian M, Salehiniya H, Afshari M. An epidemiologic study of traffic accidents on Iran drivers in 2013. Journal of Rescue \& Relief 2015; 7(1):51-60.

16- Mohammadian M, Hajari A, Mohammadian Hafshejani A. Incidence trends of injury and mortality from traffic accidents in urban and suburban areas of Isfahan province during 2002-2010. Journal of Police Medicine 2014; 3(1):1-10.

17- Akbari M, Naghavi M, Soori H. Epidemiology of deaths from injuries in the Islamic Republic of Iran. East Meditr Health J 2006; 12(34):382-90.

18- Nazari R, Bajani A, Haji Hosseini F, Beheshti $\mathrm{Z}$, Sharifnia SH, Hojati H. Mortality and injury severity in the accident victims referred to the Hefdah Shahrivar Hospital of Amol; 2007. Journal of Babol University of Medical Sciences 2011; 13(1):76-81.

19- Menon A, Pai VK, Aravindhakshan R. Pattern of fatal head injuries due to vehicular accidents in Mangalore. J Forensic Leg Med 2008; 15(2):75-7.

20- Dandona R, Kumar GA, Ameratunga S, Dandona L. Road use pattern and risk factors for non-fatal road traffic injuries among children in urban India. Injury 2011; 42(1):97103.

21- Salary A, Pirayesh Haddad F, Aghili A. Demography of trauma patients due to driving accident in Yazd city. Journal of Shahid Sadoughi University of Medical Sciences and Health Services 2002; 10(3):19-26. 
22- Karbakhsh M, Rostami Gooran N, Zargar M. Factor influencing the severity of injuries in motor vehicle crashes. Payesh Health Monitor Journal of the Iranian Institute for Health Sciences Research 2004; 3(4):273-8.

23- Swiss. World Health Organization. Department for Disability / Injury Prevention and Rehabilitation Social Change and Mental
Health Cluster. Violence and Injury Prevention Team. Injury: a leading cause of the global burden of disease. E. Krug, editor. Geneve: World Health Organization; 1999. Report No: W HO/HSC/PVI/99.11

Available from:

http://www.who.int/iris/handle/10665/66160 